\title{
Un édifice en mouvement. Projet de transformation de la Florence House à Hillbrow, Johannesburg, Afrique du Sud
}

\author{
Christophe Hutin y Daniel Estevez *
}

\section{Résumé}

Cet article collectif présente une stratégie de conception appliquée à la réhabilitation d'un immeuble de logement en contexte critique. La Florence House est un ancien hôpital désaffecté qui est aujourd'hui occupé spontanément par les habitants les plus pauvres de la ville de Johannesburg sans aucun aménagement formel. Comment des architectes peuvent intervenir dans ce contexte extrême ? Pour quoi faire ? Selon quels principes ? La stratégie défendue ici repose sur quelques principes opérationnels et théoriques simples : l'observation précise et méthodique du contexte, l'économie de moyen, la pensée du recyclage, l'utilisation des institutions informelles, la reconnaissance de toutes les compétences du milieu. Dans ce travail, l'architecte intervient moins en expert qu'en chercheur actif. II doit d'abord comprendre la vie des lieux. Avec ses interactions humaines, ses capacités matérielles, son histoire, le bâtiment prend toute l'apparence d'un milieu vivant. La démarche de l'architecte social, fondée sur la délicatesse, se rapproche alors beaucoup de celle du jardinier.

\section{Mots Clefs}

Architecture; Conception; Logement Économique; Contextualisme; Recyclage Urbain

\section{Resumen: Un edificio en movimiento. Proyecto de transformación de la Florence House en Hillbrow, Johannesburg, Sudáfrica}

El presente artículo colectivo presenta una estrategia de diseño arquitectónico en el contexto de la rehabilitación de viviendas precarias. Florence House es un antiguo hospital abandonado que ahora está ocupado, de manera informal y sin ningún tipo de planificación, por los habitantes más pobres de la ciudad de Johannesburgo. ¿Qué justificaría una intervención arquitectónica pertinente en un contexto tan extremo? ¿Para qué y en función de qué principios tendrán que hacerlo? La estrategia que aquí se defiende se basa en algunos principios simples tanto teóricos como operativos: la observación precisa y metódica del contexto, la economía, el reciclaje, el uso de las instituciones informales existentes, el reconocimiento de todas las habilidades del medio ambiente. La tarea del arquitecto debe ser menos de experto que de investigador activo, ya que debe, en primer lugar, entender la vida de los lugares. Con sus interacciones humanas, sus capacidades, su historia, el edificio asume la apariencia de un organismo vivo. El trabajo del arquitecto social, basado en la sensibilidad y la delicadeza, se parecerá mucho al del jardinero.

\section{Palabras clave}

Arquitectura; Diseño; Viviendas Asequibles; Contextualismo; Reciclaje Urbano

\section{Abstract: A building in motion. The Florence House transformation project in Hillbrow, Johannesburg, South-Africa}

This paper presents a design strategy applied to the rehabilitation of housing in critical context. Florence House is an old abandoned hospital that is now spontaneously occupied by poorest inhabitants of the city of Johannesburg without any formal planning. How architects can be involved in the improvement of such a extreme environment? In order to do what? Following which principles? The strategy advocated here is based on some simple theoretical and operational Arquitectos, profesores en la ENSA Toulouse, investigadores del LRA (Laboratorio de Investigación en Arquitectura). 
principles that are: a precise and methodical observation of context, the economy of means, the thought of recycling, the use of informal institutions, recognition of all the skills existing into the site. In this work, the architect acts more like an active researcher less rather than pure expert. He must first understand the life of the place. With its human interactions, its hardware capabilities, its history, the building takes on the appearance of a living environment. The social architect, whose work is based on discretion, then looks like a gardener.

\section{Key words}

Architecture; Design; Affordable Housing; Contextualism; Urban Recycling

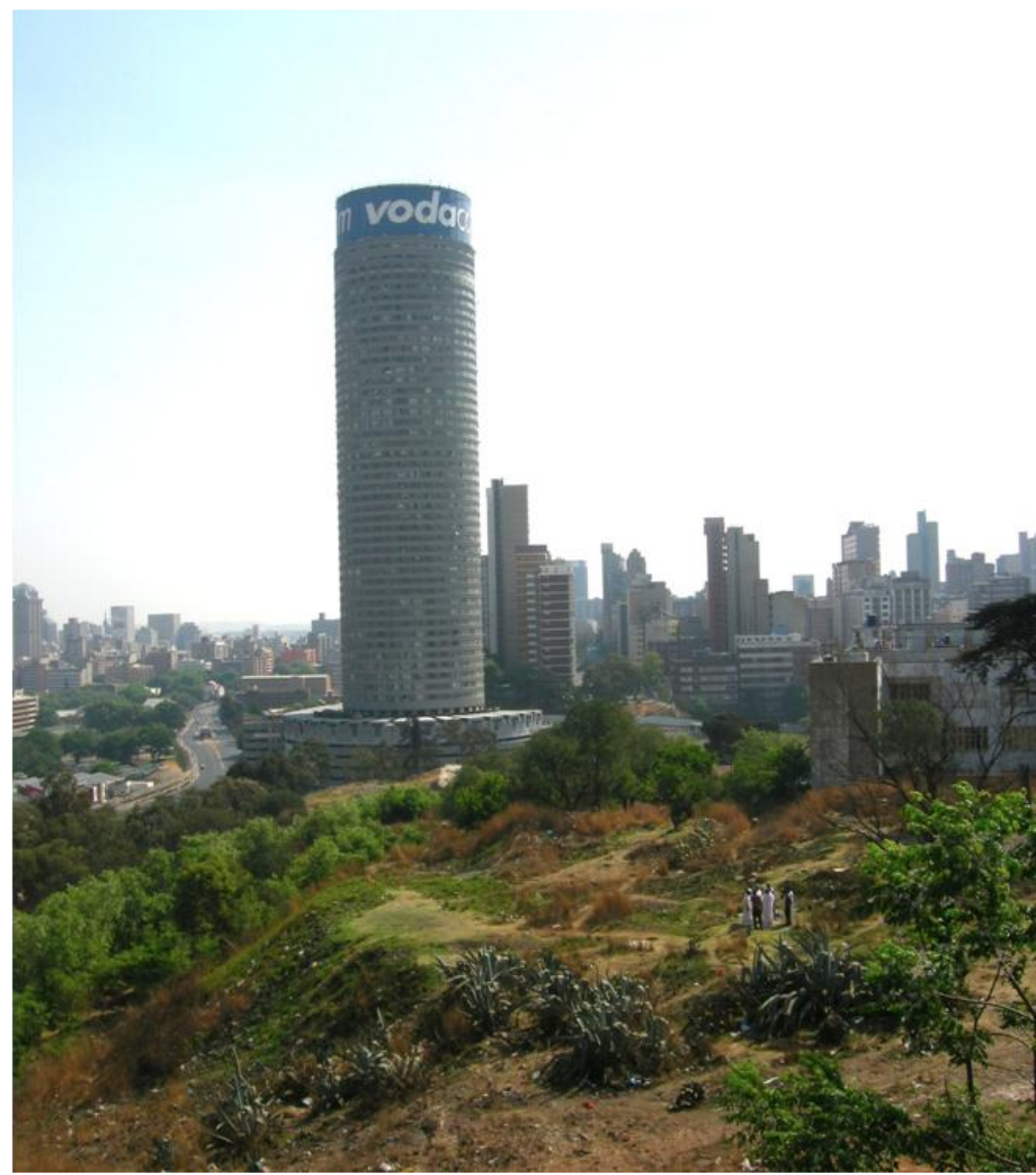

Figura 1: El barrio de Hillbrow. Fuente: Atelier Learning From (2011). 


\section{Architecture : quand le mouvement est une stratégie}

En 1991, le paysagiste Gilles Clément propose de renouveler le mode de conception des jardins en s'inspirant de la friche : un espace de vie laissé au libre développement de ceux qui s'y installent, quels qu'ils soient. Le "jardin en mouvement" qu'il propose est un espace en évolution et son développement est seulement infléchi par le jardinier mais jamais imposé.

Ici la tâche du jardinier commence par une activité d'observation, il doit interpréter précisément les interactions entre les êtres vivants, comprendre et préserver la richesse du réel, en tirer le meilleur usage. Son objectif est toujours de "maintenir et accroître la diversité, source d'étonnement, garantie du futur" (Clément, 1991) et pour cela il faut également maintenir et accroître la qualité des supports existants et donc intervenir avec la plus grande économie de moyens.

Cet état d'esprit, qui conduit le jardinier à "observer plus et jardiner moins", nous pouvons le transposer au domaine plus général de l'architecture. II est à la base du travail de projet présenté dans ce texte, qui suppose que, tout comme un jardin, une architecture n'a rien d'inerte.

II s'agit donc d'accompagner les situations pour les transformer. II s'agit d'observer, d'arpenter, d'interpréter et de donner par là une réelle pertinence à notre intervention d'architecte dans le milieu ambiant. Nettoyer, réparer, ajouter, prolonger, telles sont alors les actions fondamentales d'une architecture en mouvement. Simplicité et délicatesse, l'architecte agit et se rend invisible.

\section{Économiser}

Situé dans le quartier Hillbrow, en plein centre de Johannesburg en Afrique du Sud, la Florence House est un ancien hôpital aujourd'hui désaffecté. Comme de nombreux édifices semblables de ce quartier abandonné, le bâtiment est occupé illégalement par des centaines de familles pauvres provenant de différents pays limitrophes.

Dans ce contexte extrêmement précaire, tout projet d'amélioration appliqué depuis l'extérieur doit être mené avec prudence et évalué précisément tant il est facile de rompre les fragiles équilibres en place. Ici, pour l'architecte, il s'agit donc d'abord de réemployer ce qui existe déjà et de soutenir la nouvelle vie de l'ancien hôpital.

Pour cette raison, le principe d'économie de moyen est au centre de notre projet de réhabilitation. Michel De Certeau résume très bien cette attitude de conception : "avec le minimum de forces, obtenir le maximum d'effets. La multiplication des effets par la raréfaction des moyens est, pour des raisons différentes, la règle qui organise à la fois un art de faire et l'art poétique." (De Certeau, 1990).

Si les dispositifs adoptés par le projet paraissent souvent modestes, ils recherchent toujours cependant ce que l'on pourrait nommer une puissance, c'est à dire une efficience, une influence pertinente sur le milieu. Rechercher la pertinence, tel est l'objectif premier.

Aménager un marché ouvert temporaire sur un parking, ajouter des portes à certains endroits précis des couloirs, remettre en service une entrée existante abandonnée du bâtiment et pourtant névralgique, équiper un escalier, réemployer des conduits désaffectés, modifier le cloisonnement de certaines pièces, ajouter une terrasse accessible, nettoyer, réparer... tout cela ne constitue pas des gestes irréversibles. Ce sont des interventions souples, et cette multiplicité d'actions est presque imperceptible dans le dessin des plans du bâtiment.

Pour autant, tous ces dispositifs d'économie ne constituent en rien une accumulation de détails. Ils répondent au contraire à une stratégie de projet, celle du mouvement dont parle Gilles Clément. Agir sur les multiples points d'appui de 
l'existant et le transformer. Agir avec justesse pour infléchir la pratique actuelle de l'édifice par les habitants, plutôt qu'imposer des formes préconçues.

\section{Institutions spontanées et espaces en commun}

Le processus de réhabilitation de la Florence House a commencé par l'identification des institutions crées par les habitants : une crèche au deuxième étage, une chapelle improvisée au sous sol, une école de musique... il s'agissait d'abord de conforter leur existence. Le terme institution est pris ici dans le sens de dynamique collective. Il doit être rapproché des réflexions du pédagogue Fernand Oury qui définit cette notion d'institution comme "un système de médiation dans lequel les personnes ne sont plus simplement face à face, mais parlent de quelque chose qui existe et œuvrent sur quelque chose qui existe en dehors d'eux et dont ils sont responsables." (Oury, 1998). Dans notre projet, les espaces communs du bâtiment sont donc, autant que possible, considérés comme des institutions, c'est à dire des supports de coopération et de partage. Certains de ces lieux, comme les ateliers ou les laveries, n'existent pas formellement dans l'état actuel de la Florence, ils seront créés conformément aux besoins qui ont été exprimés ou repérés au cours des rencontres et des visites. Mais en tout état de cause, leur conception s'appuie sur les compétences des habitants à faire vivre ces microinstitutions, qu'elles soient existantes ou potentielles.

\section{Communauté : diviser l'espace pour réunir les habitants}

En découvrant l'état actuel de la Florence House, on est d'abord frappé par le caractère labyrinthique du bâtiment. La multitude d'entrées, de couloirs d'accès et de cages d'escaliers donne une impression de complexité. En fait, comme tout hôpital, la Florence est constituée d'un système de circulations redondantes liées aux contraintes spécifiques du soin : accès aux chambres, déplacement des patients, circulation des brancards, distributions du linge, de la nourriture, rejet des déchets, etc. (fig. 4). Pourtant, si cette abondance de dispositifs de distribution est réelle, elle constitue aussi une véritable richesse spécifique de ce lieu et permet d'envisager nombre de combinaisons pour une réinvention du bâtiment sur lui-même.

Mais dans la Florence House squattée, la peur constante de l'éviction amène les habitants à éprouver une certaine crainte de l'extérieur. Hors des murs de la Florence, bien sûr, mais aussi dans le bâtiment lui-même, les habitants ne se sentent pas toujours en sécurité ; en témoignent les larges couloirs souvent déserts ou bien ces familles voisines qui ne se connaissent pas et n'entretiennent parfois aucune relation.

Dans ce contexte, la multiplication des accès et des circulations rend actuellement l'édifice perméable à tous les passages. Cette perméabilité entraîne des inquiétudes. II est difficile par exemple de laisser jouer des enfants dans le bâtiment sans surveillance si tout couloir est un lieu aussi public qu'une rue. Cette réalité tend à renvoyer chaque habitant sur l'espace clos de son propre appartement. Comment dès lors maintenir une richesse de circulation propre à favoriser les déplacements et les voisinages tout en réduisant la perméabilité inquiétante de l'édifice ?

La stratégie choisie a consisté à redéfinir des unités de voisinages intermédiaires en vue de renforcer la notion de communauté dans le bâtiment (fig. 5). La communauté est en effet une donnée importante de la structure sociale des quartiers populaires de Johannesburg. Pour nous, elle définit un critère de pertinence du projet : notre travail doit s'appuyer sur ces structures invisibles sans quoi nos propositions ne seront 
tout simplement pas reçues par les habitants.

Ainsi, en s'appuyant sur les quatre principaux escaliers existants, le projet propose une division sociale de l'ensemble de la Florence en quatre quartiers de taille à peu près équivalente. Cette division est opérée par des éléments de second œuvre et n'entraîne pas de modification de la structure bâtie du bâtiment.
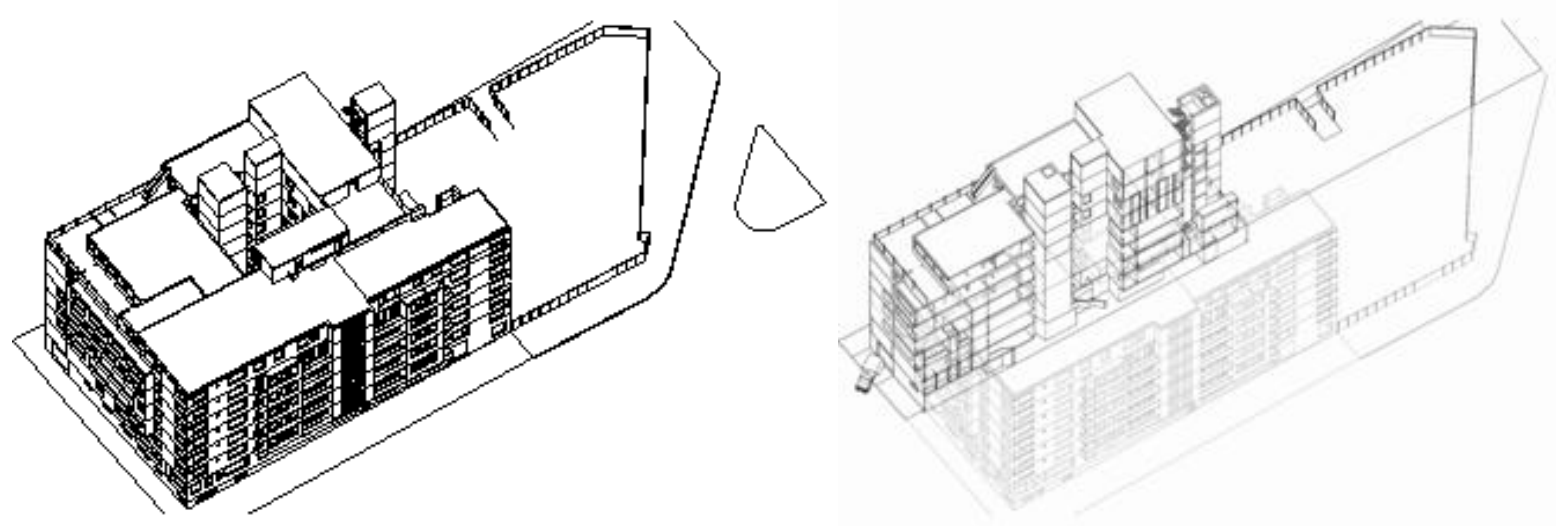

Figuras 2 y 3. Florence House, anatomía del proyecto. Fuente: Atelier Learning From (2011).
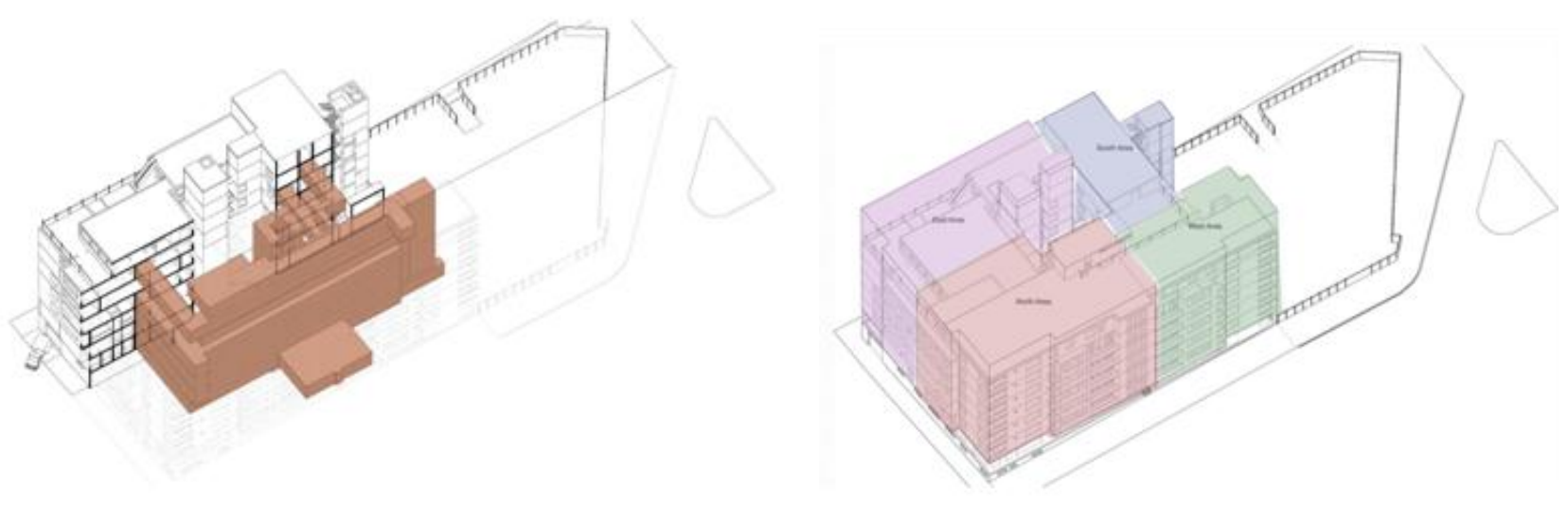

Figuras 4 y 5. Florence House, anatomía del proyecto. Fuente: Atelier Learning From (2011).

\section{Équiper les unités de voisinage, faciliter la vie quotidienne}

Les quatre secteurs indépendants, desservis par des escaliers distincts (fig. 7) seront réalisés à l'intérieur du bâtiment au moyen de simples portes de séparation translucides qui assureront le maintien d'une transparence tout en clarifiant le passage d'une zone à une autre. Pour renforcer l'identité de chacun des quatre quartiers, le projet prévoit également d'y ajouter certains éléments de programme spécifiques, propres à donner une qualité particulière à chacun des secteurs. Ainsi, le quartier Nord possèderait des logements traversants, le quartier Est offrirait un dortoir pour personnes en transit, Le quartier Sud une laverie collective, etc.

En divisant les étages courants par quartier, chaque sous-ensemble d'habitations peut être associé de façon logique à son système de pièces de service communes : laverie, sanitaires, vide ordure.

Les conduits de gaines et de monte-charge divers sont très nombreux dans un hôpital. Ici, ces dispositifs désaffectés sont disponibles pour un 
nouvel usage, ils constituent une ressource fonctionnelle. En particulier ils permettent de proposer des colonnes de vide-ordures assez amples pour ne pas être facilement bouchées. (Fig.6). Grâce à ces conduits, les ordures ménagères peuvent être collectées dans des bennes situées au sous-sol, niveau accessible aux camions pour l'évacuation. La mise en place et le remplacement de ces bennes collectives seraient ainsi assurés au niveau de l'ensemble de la Florence House. Les vide-ordures simplifient la gestion quotidienne des déchets dans le bâtiment et ses quartiers, ils minimisent le transport individuel des ordures ; cette question n'est pas négligeable pour le confort des occupants car le bâtiment n'est équipé d'aucun ascenseur et tous les déplacements se font à pied.
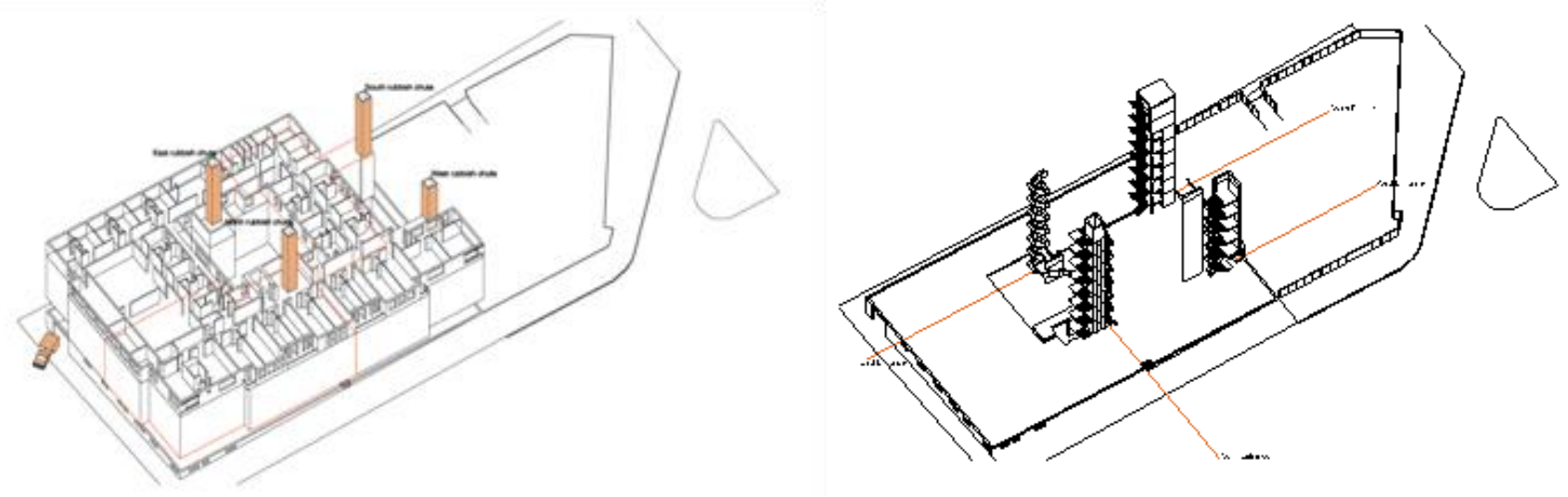

Figura 6 y 7. Florence House, anatomía del proyecto. Fuente: Atelier Learning From (2011).

\section{L'eau}

L'observation des usages existants autour de l'eau, du lavage du linge et des laveries improvisées a aussi permis de dégager des propositions architecturales particulières. Ce qui était intéressant c'est que nous avons pu connecter cette observation avec un témoignage recueilli dans le squatter camp de Kliptown à Soweto où un habitant avait expliqué : "Every house that you see when you look around, they all come here for water, they all collect their water here. This is just more than a tap or more than a story about this tap. Such instruments are the one that manage to put us together. You know, I mean you'll find out ten people coming at the same tap at the same time. That's where conversation starts, that's where relationship starts. That's where people get to know each other more better." ${ }^{1}$.

\footnotetext{
${ }^{1}$ Témoignage présenté sur le blog de l'Atelier Learning From (Estevez y Hutin, 2011).
}

Le fait que le système de distribution de l'eau soit un outil de sociabilité pour la communauté des habitants, est un de ces points d'appui que nous recherchons pour soutenir la pertinence du projet. Des propositions ont donc été faites pour valoriser les pièces collectives destinées à l'eau, au lavage et au séchage du linge etc. Actuellement les endroits communs destinés au séchage sont parmi les plus agréables dans le bâtiment, ils sont bien ventilés, en particulier au dernier étage, souvent couvert ou pouvant posséder un bon ensoleillement.

Avec ce travail, il ne s'agit donc pas seulement de proposer des pièces de services réparties dans les étages selon les quatre quartiers de la Florence. Leur réhabilitation et le soin apporté à leur réparation et à leur confort (dimensions, ventilations, éclairement) constitue en fait un acte de valorisation sociale des pratiques quotidiennes. 


\section{Rendre l'espace plus équitable}

La Florence House est un lieu hors du droit légal où les règles sont imposées par un système spontané. Ce tissu social complexe est basé sur des données qui ne sont connues que de ses habitants et qu'il est difficile de se représenter sans une réelle immersion dans les lieux. Si l'on recoupe les informations et témoignages, que nous avons recueillis, les tensions sont nombreuses, elles naissent pour différentes raisons mais touchent souvent à des questions d'équité entre les occupants. II suffit d'entendre les paroles de ces habitants pour comprendre que l'injustice se ressent.

Dans ce contexte incertain et parfois conflictuel, notre rôle est d'éviter que l'espace soit le vecteur d'un sentiment d'injustice. II s'agit de permettre au contraire que le logement puisse procurer d'autres sentiments, qu'il contribue à la pacification des relations sociales et favorise une plus grande équité. Dans le cas de la Florence House, la création d'un tissu spontané, cousu et improvisé

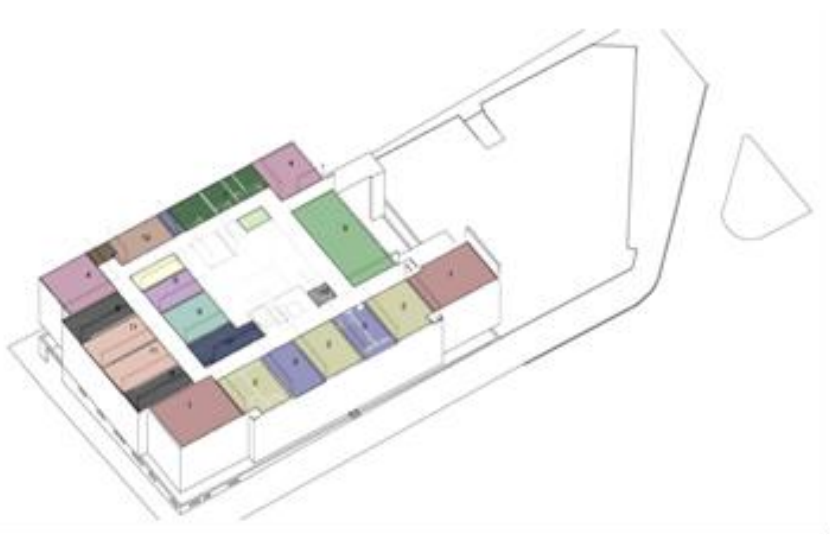

au jour le jour a créé un rapport du nombre d'habitant par mètre carré irrationnel et non équitable. La recherche de solutions à ces situations s'est tout d'abord appuyée sur l'analyse précise de la structure constructive du bâtiment en distinguant sa partition primaire permanente et ses subdivisions secondaires temporaires.

La structure primaire constitue le squelette porteur du bâtiment et définit les partitions fondamentales des étages courants qu'il n'est pas possible de modifier facilement. Un inventaire des morphologies d'espace données par cette structure a permis de distinguer 17 entités spatiales de base par étage, (fig. 8) cela définit la capacité globale du bâtiment. A partir de là, différentes entités présentant une même morphologie peuvent être divisées de façon différente en fonction des besoins d'espace de chaque habitant. Ces différents types de sous-cloisonnement intègrent également la réhabilitation de pièces de services sanitaires affectées à chaque entité d'habitation.

Figura 8. Florence House, anatomía del proyecto. Fuente: Atelier Learning From (2011).

\section{Un projet structuraliste}

A chaque étage, les partitions secondaires sont donc des configurations de cloisonnement plus ou moins temporaires. Dans l'état actuel de la Florence House, ces subdivisions résultent de décisions spontanées des habitants. On peut dire qu'elles sont issues de la performance des occupants, tandis que les partitions primaires relèvent quant à elles des capacités fixes, ou compétences, du bâtiment. Cette distinction est fondamentale, nous devons la préserver dans le projet. Les cloisonnements de second œuvre doivent pouvoir être modifiés sans entraîner des déplacements des éléments de services, salles de bains, cuisines, sanitaires.

Ainsi on peut envisager que la transformation de l'architecture dans le temps résulte des appropria- 
tions variables d'une structure fixe : "en termes architecturaux, la compétence est la capacité de la forme à être interprétée, et la performance est la manière dont la forme est interprétée." (Hertzberger, 2009). Cette métaphore linguistique citée par Herman Hertzberger a souvent été reprise. Elle signifie que la vie sociale abritée par l'architecture est un phénomène volubile et instable qui doit pourtant être préservé comme tel. C'est aussi le sens des réflexions de Michel De Certeau, dans son livre L'invention du quotidien : "Seule une langue morte ne se modifie plus, seule l'absence de tout résident respecte l'ordre immobile des choses. La vie entretient et déplace, elle use, casse et remanie, elle crée de nouvelles configurations d'êtres et d'objets, à travers les pratiques quotidiennes des vivants, toujours semblables et différents." (De Certeau, 1990).

\section{L'inventaire, un outil pour projeter à partir du contexte}

Pour un architecte, réaliser un projet en site occupé présente le précieux intérêt de permettre une rencontre avec les habitants qui y vivent.

Au sein de la Florence House, les habitants actuels vivent dans un équilibre économique et social précaire. Immigration, travail informel, absence de ressources concernent la plupart des gens et dans ce contexte toute action brutale de rénovation du bâtiment risque de rompre les équilibres des communautés en place. Travailler autant que possible sans que les habitants aient à déménager est donc une nécessité absolue.

Au cours des rencontres avec les habitants il a fallu parvenir à une connaissance qualitative et précise des besoins de chaque famille en évitant l'approche strictement quantitative et statistique. II s'agissait en particulier de savoir combien de personnes vivaient dans chaque logement, quelles étaient leurs relations familiales et amicales, leurs projets. A travers ces échanges, on pouvait comprendre que la notion de famille est ici étendue aux amis dont la présence constitue souvent un soutien aussi fort que celui découlant d'une parenté stricte.

Un inventaire exhaustif des pièces habitées, réalisé au cas par cas, a été dressé collectivement par l'atelier. II a fait apparaître trois situations récurrentes : la sur-occupation de certains logements (une famille de cinq personnes se partageant une surface de $13 \mathrm{~m} 2$ par exemple), la sous-occupation relative d'autres (une personne seule vivant dans $22 \mathrm{~m} 2$ par exemple) et des personnes d'une même famille répartie, faute d'espace, dans plusieurs logements. En recherchant à remédier à ces déséquilibres, un plan d'occupation alternatif de la Florence s'est dégagé avec une proposition d'échanges de logements entre habitants au sein d'un même étage de la Florence House.

Pour que cette proposition soit réalisable et constitue un véritable gain pour les habitants, les mouvements internes doivent être accompagnés d'une révision partielle des cloisonnements secondaires de l'étage courant. II s'agit donc de repenser cette partition au regard des nouveaux besoins décelés : davantage d'appartements pour personnes célibataires, quelques logements permettant d'accueillir des familles nombreuses ou encore un dortoir indépendant par étage permettant aux personnes en transit d'être logés dans la Florence.

Réaffectations, rapprochements, répartitions, tous ces mouvements internes visent à consolider la dynamique de vie du bâtiment.

\section{L'hospitalité}

Aujourd'hui la Florence House est de fait un lieu de passage et de brassage de différentes populations. En plus de ses habitants quasi permanents, on rencontre un grand nombre de personnes qui ne sont installées que temporairement. Ainsi le squat fournit une aide concrète et joue dans cer- 
tains cas le rôle de relai ou de maison-étape auprès de personnes en transit. Ce rôle peut être encouragé par notre intervention.

Actuellement, la parcelle de la Florence House est divisée en son milieu en deux parties équivalentes en surface, sur l'une se trouve le bâti de l'ancienne maternité tandis que l'autre, à l'ouest, contient actuellement un parking qui pourrait être réaménagé en marché ouvert. Sur cette surface libre, accolée à la façade ouest du bâtiment, on propose d'insérer une petite structure à ossature acier de deux étages. II s'agit de la seule construction neuve du projet, elle est reliée à la zone nord-ouest et à l'actuel parking (futur marché) par son accès extérieur.

Cette extension sera aménagée en huit logements d'hébergement temporaire. Dans un premier temps, ces logements seront occupés à tour de rôle par des habitants de la Florence House dont les habitations sont impactées momentanément par les travaux de réhabilitation et nécessitent un déménagement temporaire. Le fait de projeter en site occupé nous contraint en effet à trouver une alternative d'accueil afin d'éviter le déménagement des habitants hors de la parcelle. La proximité de ce gîte d'hébergement, situé de plain pied avec les logements initiaux, simplifiera les déménagements.

Une fois les travaux de la Florence House effectués, ces nouveaux logements pourront accueillir des personnes en transit et conserver son rôle d'institution d'hospitalité.

\section{Referencias}

CLÉMENT, Gilles. Le Jardin en Mouvement. Paris: Sens et Tonka, 1991.

DE CERTEAU, Michel. L'invention du quotidien 1. Arts de faire. Paris: Folio Essai, Gallimard, 1990. ESTEVEZ, Daniel y HUTIN Christophe. Where conversations start. Learning from, ENSAToulouse, 14 de enero de 2011. <http://learning- from.over-blog.fr/article-where-conversationsstart-64992270.html>. Consultado el 4 de febrero de 2013).

ERTZBERGER, Herman, Leçons d'architecture. Paris: Edition In Folio, 2009.

OURY, Fernand. Vers une pédagogie institutionnelle ?. Paris : Vigneux, Matrice, 1998.

\section{Cita del artículo}

HUTIN, C., ESTEVEZ, D. Un édifice en mouvement. Projet de transformation de la Florence House à Hillbrow, Johannesburg, Afrique du Sud. Hábitat y Sociedad, 2012, no 5, p. 123-131.

$<$ www.habitatysociedad.us.es $>$.

http://dx.doi.org/10.12795/HabitatySociedad.2012.i5.09 